ARTICLE

Received 5 May 2014 | Accepted 27 Jun 2014 | Published 28 Jul $2014 \quad$ DOl: 10.1038/ncomms5555

\title{
High-temperature superfluidity with indirect excitons in van der Waals heterostructures
}

M.M. Fogler ${ }^{1}$, L.V. Butov ${ }^{1} \&$ K.S. Novoselov ${ }^{2}$

All known superfluid and superconducting states of condensed matter are enabled by composite bosons (atoms, molecules and Cooper pairs) made of an even number of fermions. Temperatures where such macroscopic quantum phenomena occur are limited by the lesser of the binding energy and the degeneracy temperature of the bosons. High-critical temperature cuprate superconductors set the present record of $\sim 100 \mathrm{~K}$. Here we propose a design for artificially structured materials to rival this record. The main elements of the structure are two monolayers of a transition metal dichalcogenide separated by an atomically thin spacer. Electrons and holes generated in the system would accumulate in the opposite monolayers and form bosonic bound states-the indirect excitons. The resultant degenerate Bose gas of indirect excitons would exhibit macroscopic occupation of a quantum state and vanishing viscosity at high temperatures.

\footnotetext{
${ }^{1}$ Department of Physics, University of California, San Diego, 9500 Gilman Drive, La Jolla, California 92093, USA. ${ }^{2}$ School of Physics and Astronomy, University of Manchester, Manchester M13 9PL, UK. Correspondence and requests for materials should be addressed to M.M.F.

(email: mfogler@ucsd.edu).
} 
C oherent states of excitons have been a subject of intense theoretical studies ${ }^{1-3}$. A general framework for creation and manipulation of degenerate gases of indirect excitons has been established in prior experimental studies of GaAs-based coupled quantum wells (CQWs) where electrons and holes are confined in GaAs quantum wells separated by a thin AlGaAs barrier ${ }^{4,5}$. Here we apply similar principles to the design of a CQW from atomically thin materials stacked on top of each other. Research on such van der Waals heterostructures is gaining momentum in the last few years ${ }^{6}$, and their quality and availability is steadily improving.

In the proposed device (Fig. 1a), an indirect exciton is composed from an electron and a hole located in two different $\mathrm{MoS}_{2}$ layers separated by a hexagonal boron nitride (hBN) insulating barrier and surrounded by $\mathrm{hBN}$ cladding layers. The $z$-direction electric field is controlled by voltage applied to external electrodes. The applied field modifies the band structure in a way that it becomes advantageous for optically excited electrons and holes to reside in the opposite $\mathrm{MoS}_{2}$ monolayers and form indirect excitons (Fig. 1b).

The design of the $\mathrm{MoS}_{2} / \mathrm{hBN}$ structure is similar to that of GaAs/AlGaAs CQW we studied previously ${ }^{4,5}$, except GaAs is replaced by $\mathrm{MoS}_{2}$ and AlGaAs by hBN. Here we predict the phase diagram of such a device using the results of numerical calculations and scaling arguments. Our most intriguing finding is that in the proposed $\mathrm{MoS}_{2} / \mathrm{hBN}$ structures degenerate Bose gas of indirect excitons can be realized at record-high temperatures.

\section{Results}

Degeneracy temperature. The characteristic temperature $T_{d}$ at which excitons become degenerate is determined by their density $n_{\mathrm{x}}$ per flavour (spin and valley), and effective mass $m_{\mathrm{x}}=m_{\mathrm{e}}+m_{\mathrm{h}}$ :

$$
k T_{\mathrm{d}}=\frac{2 \pi \hbar^{2}}{m_{\mathrm{x}}} n_{\mathrm{x}} .
$$

Here $m_{\mathrm{e}}$ and $m_{\mathrm{h}}$ are the electron and hole effective masses. For $n_{\mathrm{x}} \sim 10^{10} \mathrm{~cm}^{-2}$, and $m_{\mathrm{e}}=0.07, m_{\mathrm{h}}=0.15, m_{\mathrm{x}}=0.22$ representative of GaAs CQW, we find $T_{\mathrm{d}} \sim 3 \mathrm{~K}$. (Here and below all the masses are in units of the bare electron mass.) At such temperatures long-range spontaneous coherence of indirect excitons is observed ${ }^{5}$.

To explain why $\mathrm{MoS}_{2}$-based CQW would possess much higher $T_{\mathrm{d}}$ than GaAs-based ones, we consider the characteristic length

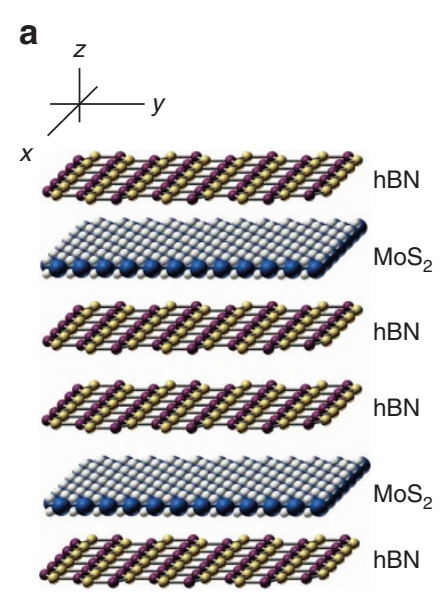

b

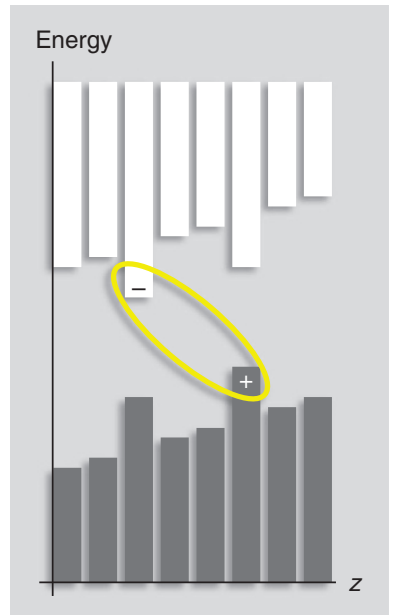

Figure 1 | Schematics of the proposed device. (a) Geometry and (b) band structure. The ellipse indicates an indirect exciton composed of an electron $(-)$ and a hole $(+)$. and energy scales in the problem, which are the exciton Bohr radius $a_{\mathrm{x}}=\hbar^{2} \epsilon_{1} / \mu e^{2}$ and the Rydberg energy $\mathrm{Ry}_{\mathrm{x}}=\hbar^{2} / 2 \mu a_{\mathrm{x}}^{2}$ defined in terms of reduced mass $\mu=m_{\mathrm{e}} m_{\mathrm{h}} / m_{\mathrm{x}}$. For estimates we use the effective static dielectric constant $\epsilon_{1}=4.9 \mathrm{of} \mathrm{hBN}$. We also use the calculated value $\mu=0.25$ (refs 7,8) for $\mathrm{MoS}_{2}$. On the basis of the measurements ${ }^{9}$ done on a related compound $\mathrm{MoSe}_{2}$, this value should be accurate to within $20 \%$. We find $a_{\mathrm{x}} \approx 1.0 \mathrm{~nm}$, much shorter than $15 \mathrm{~nm}$ in GaAs CQW. In turn, $\mathrm{Ry}_{\mathrm{x}} \approx 140 \mathrm{meV}$ in the proposed device, about forty times larger than in GaAs.

Since it enters the denominator of equation (1), one may think that having larger $m_{\mathrm{x}}$ is unfavourable for attaining higher $T_{\mathrm{d}}$. In fact, when $n_{\mathrm{x}}$ can be controlled, the opposite is true. Indeed, equation (1) can be rewritten as

$$
k T_{\mathrm{d}}=4 \pi\left(m_{\mathrm{e}} m_{\mathrm{h}} / m_{\mathrm{x}}^{2}\right)\left(n_{\mathrm{x}} a_{\mathrm{x}}^{2}\right) \mathrm{Ry}_{\mathrm{x}} .
$$

The upper limit on $n_{\mathrm{x}} a_{\mathrm{x}}^{2}$ is imposed by quantum dissociation of excitons that occurs when the ratio of the exciton size (the inplane gyration radius) $r_{\mathrm{x}}$ and the mean inter-exciton distance $1 / \sqrt{n_{\mathrm{x}}}$ reaches the critical value ${ }^{10-12}$ of about 0.3 . In the case of our primary interest where interlayer centre-to-centre distance $c \approx a_{\mathrm{x}}$ and $m_{\mathrm{e}}=m_{\mathrm{h}}=2 \mu$, we estimate $r_{\mathrm{x}}=2.4 a_{\mathrm{x}}$ (Fig. 2 ), so that the corresponding Mott critical density $n_{\mathrm{M}}$ is set by the condition

$$
n_{\mathrm{M}} a_{\mathrm{x}}^{2} \sim 0.02 \text {. }
$$

Substituting this into equation (2), we obtain

$$
k T_{\mathrm{d}}^{\max } \sim 0.06 \mathrm{Ry}_{\mathrm{x}} .
$$

Hence, the key to high $T_{\mathrm{d}}$ is the enhanced value of $R y_{\mathrm{x}}$. The exciton binding energy $E_{\text {ind }} \approx 0.6 \mathrm{Ry}_{\mathrm{x}}$ (Fig. 2) does not pose further fundamental limitations. Actually, the conclusion that the theoretical maximum of $T_{\mathrm{d}}$ is proportional to $\mathrm{Ry}_{\mathrm{x}}$ follows from dimensional analysis. If $c \sim a_{\mathrm{x}}, m_{\mathrm{e}} \sim m_{\mathrm{h}}$ and excitons are treated as an equilibrium Bose gas, then $\mathrm{Ry}_{\mathrm{x}}$ is the only relevant energy scale in the problem.

In reality, only quasi-equilibrium state is possible because of exciton recombination, and so limitations posed by the finite exciton lifetime $\tau$ must be discussed. Radiative recombination of indirect excitons requires interlayer tunnelling. The rate of this process can be made exponentially small by adjusting the barrier

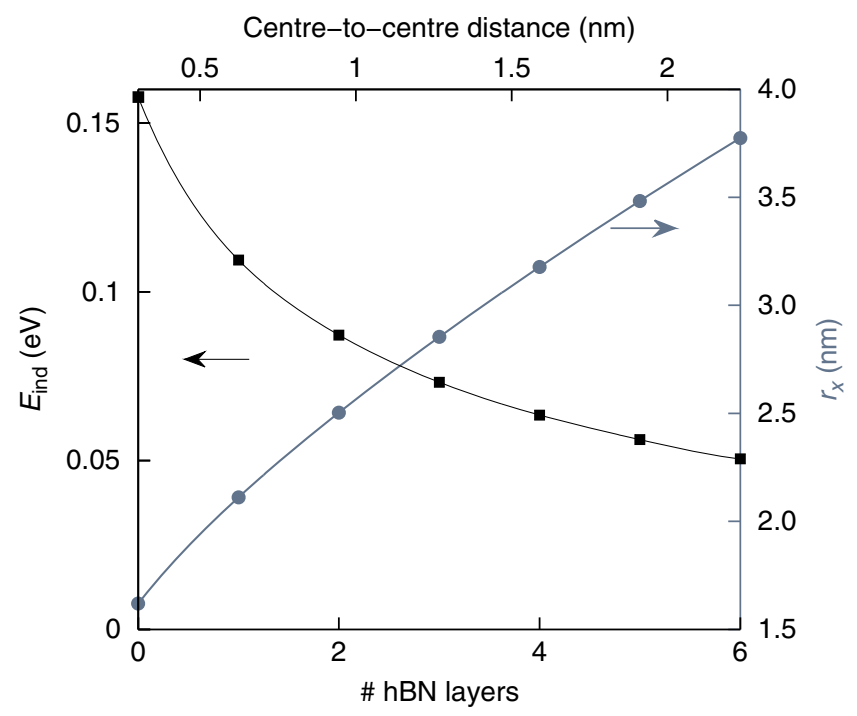

Figure 2 | Estimated exciton parameters. $E_{\text {ind }}$ (left axis) is the binding energy and $r_{\mathrm{x}}$ (right axis, equation (13)) is the in-plane gyration radius of indirect excitons. The bottom axis is the number of the hBN spacer layers and the top axis is the corresponding centre-to-centre distance between the $\mathrm{MoS}_{2}$ layers in the heterostructure. 
width. In GaAs CQW, $\tau$ can be routinely made in the range of microseconds and can be controllably varied over several orders of magnitude. The large $\tau$ enables creation of indirect exciton gases of high-density $n_{\mathrm{x}}=P \tau$ with low optical excitation power $P$ that does not cause overheating of either the lattice or the excitons. This is why for the task of achieving cold dense exciton gases, indirect excitons are superior to conventional bulk excitons or two-dimensional (2D) direct excitons (bound states of electrons and holes in the same layer).

In the proposed device, the interlayer tunnelling rate would decay exponentially with the number $N$ of layers in the hBN spacer. For $N=2$, the tunnelling rate is comparable to that in the $\mathrm{GaAs} \mathrm{CQW}^{4,5}$ (see Methods) indicating that long-life indirect excitons can be realized in $\mathrm{MoS}_{2} / \mathrm{hBN}$ structures. The tunnellinglimited lifetime can be enhanced by possible rotational misalignment of the $\mathrm{MoS}_{2}$ layers, which makes the excitons indirect not only in real but also in momentum space, similar to excitons in $\mathrm{MoS}_{2}$ multilayers ${ }^{9,13}$. Hence, sufficiently long $\tau$ may perhaps be achieved with a monolayer hBN spacer. Working with very small $N$, one has to worry about possibility of dielectric breakdown of the hBN spacer. To make the indirect exciton more energetically favourable than the direct one, a voltage equal or larger than $\left(E_{\mathrm{dir}}-E_{\mathrm{ind}}\right) / e$ must be applied between the $\mathrm{MoS}_{2}$ layers. Assuming the direct exciton binding energy $E_{\mathrm{dir}}$ of approximately ${ }^{8,14} 0.5 \mathrm{eV}$, for $N=2 \mathrm{hBN}$ spacer, the required voltage is about $0.4 \mathrm{~V}$, which is safely below the breakdown limit ${ }^{15}$.

A schematic phase diagram of a neutral electron-hole system in the proposed heterostructure is shown in Fig. 3. The solid line represents the Mott transition. It emanates from the $T=0$ critical point computed according to equation (3). At the transition there is a discontinuous jump in the degree of exciton ionization ${ }^{16}$. Above the Mott critical temperature $T_{M}$ the transition changes to a smooth crossover. Such a crossover has been studied experimentally in photo-excited single-well GaAs and InGaAs structures ${ }^{17,18}$. We assume that $T_{\mathrm{M}}$ should be lower than $T_{\mathrm{d}}^{\max }$ and use $k T_{\mathrm{M}}=0.5 k T_{\mathrm{d}}^{\max } \sim 0.03 \mathrm{Ry}_{\mathrm{x}}$ in Fig. 3 for illustration. The quantum degeneracy line (equation 1) shown by the dasheddotted line in Fig. 3 demarcates a crossover from classical to

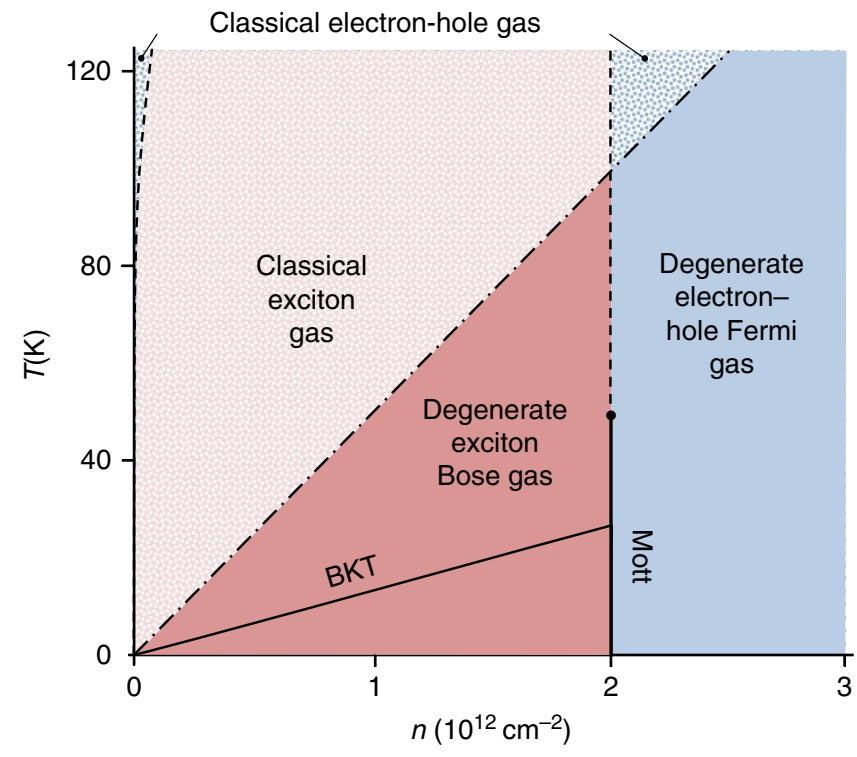

Figure 3 | Schematic phase diagram of the system studied. $T$ is the temperature and $n$ is the electron density per flavour. The dashed lines indicate crossovers and the solid lines mark the phase transitions. The dot is the Mott critical point. degenerate Bose gas of excitons. At $n=n_{\mathrm{M}}$ this line extrapolates to the temperature $k T_{\mathrm{d}}^{\max }$ (equation 4).

Whereas the Mott transition represents quantum dissociation of excitons, the thermal dissociation occurs above the Saha temperature $k T_{\mathrm{S}} \sim\left(\pi \hbar^{2} n_{\mathrm{x}} / m_{\mathrm{x}}\right) e^{E_{\text {ind }} / k T_{\mathrm{S}}}$, which is shown by the dashed line near the $T$ axis in Fig. 3. This line marks a crossover from the exciton phase to a classical plasma. Since their binding energy is very large, indirect excitons in $\mathrm{MoS}_{2} / \mathrm{hBN}$ structure remain stable against the thermal dissociation well above the room temperature in a broad range of electron densities.

Formation of quantum degenerate Bose gas of long-lifetime repulsively interacting indirect excitons leads to local exciton superfluidity below $T_{\mathrm{d}}$. The superfluidity spreads over a macroscopic area at the Berezinskii-Kosterlitz-Thouless transition temperature ${ }^{19}$

$$
k T_{\mathrm{BKT}} \approx 1.3 \frac{\hbar^{2} n_{\mathrm{x}}}{m_{\mathrm{x}}}
$$

Superfluid transport of neutral indirect excitons produces dissipationless charge currents in the opposite directions in the two layers ${ }^{2}$. To observe and utilize this effect, one can, for example, make separate contacts to each layer and form a closed circuit for the hole layer. The electric current in the electron layer will then be dissipationless. This is referred to as the counterflow superconductivity ${ }^{20}$.

The most intriguing conclusion we draw from Fig. 3 is that in the proposed $\mathrm{MoS}_{2} / \mathrm{hBN}$ structures degenerate Bose gas of indirect excitons can be realized at record-high temperatures.

\section{Discussion}

In the remainder of this paper, we overview phenomena analogous to a coherent state of indirect excitons in other $2 \mathrm{D}$ systems. We choose not to survey systems where electrons and holes reside in the same layer, such as quantum wells in microcavities ${ }^{21}$. They are interesting in their own right but counterflow superconductivity therein is impossible.

First, evidence for broken-symmetry phases conceptually similar to condensates of indirect excitons have been reported in GaAs $\mathrm{CQW}^{22}$. These phases arise in the presence of a quantizing magnetic field at sub-Kelvin temperatures. Next, a number of broken-symmetry states, some of which are similar to exciton condensates, have been predicted to form in bilayer graphene ${ }^{23}$. The double-layer graphene (DLG) systems must be specially mentioned because a high-temperature coherent state in DLG was theoretically discussed recently. The fundamental obstacle to exciton condensation in DLG is that monolayer graphene is a zero-gap semimetal with a linear quasiparticle dispersion. Although in such a system phases of weakly bound excitons may exist, the corresponding critical temperature is extremely sensitive to the effective strength of the electron-hole attraction. Calculation of the latter requires accounting for screening of the long-range Coulomb interaction as well as short-range correlation effects, both of which are challenging problems. Theoretical estimates of the critical temperature in DLG range from hundreds of Kelvin ${ }^{24-26}$ to a few milli-Kelvin ${ }^{27}$. We wish to stress that there is no room for such an enormous uncertainty in our proposal based on $\mathrm{MoS}_{2}$, a semiconductor with a significant bandgap, modest dielectic constant and exceptionally stable exciton state. The dependence of the characteristic temperatures $T_{\mathrm{d}}$ and $T_{\mathrm{BKT}}$ on electron density is linear rather than exponential. All pertinent numerical factors are constrained by numerous prior studies of similar semiconducting systems. Therefore, the error in the estimated temperatures should be small for the system considered in our paper. 


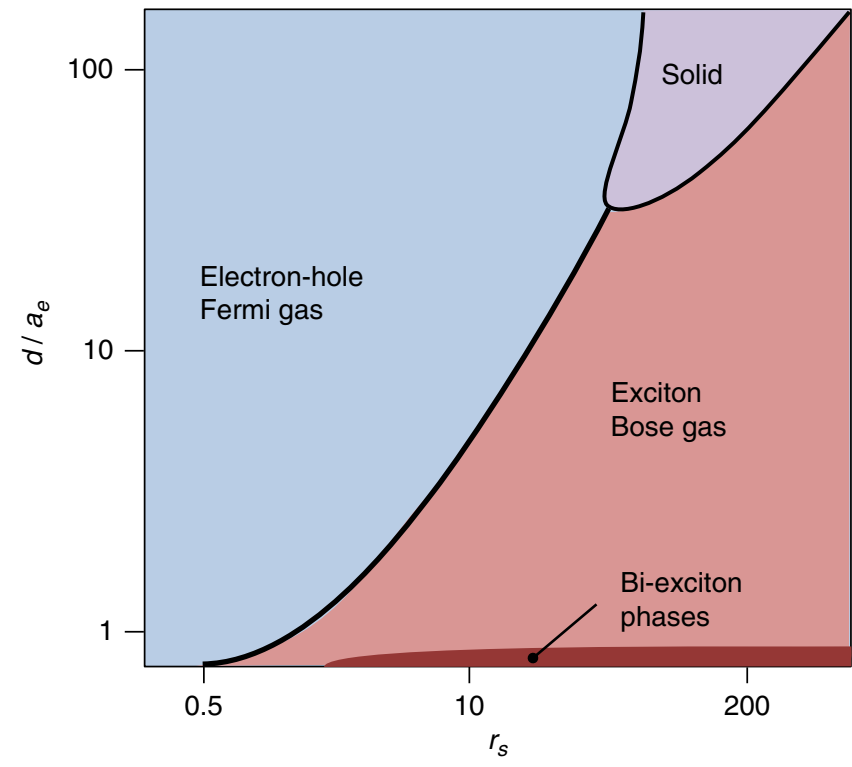

Figure 4 | Schematic $\boldsymbol{T}=\mathbf{0}$ phase diagram. The system is an electron-hole bilayer in which the particles have spin $1 / 2$ but no valley degeneracy. The $\mathrm{MoS}_{2} / \mathrm{hBN}$ device with the $N=2$ layer $\mathrm{hBN}$ spacer corresponds to $d / a_{\mathrm{e}} \approx 2.8$. At such $d / a_{\mathrm{e}}$ the ground state of the system at $r_{\mathrm{s}} \gtrsim 6$ is the superfluid Bose gas of indirect excitons.

The scaling law of equation (4) elucidates a general principle for realizing high-temperature coherent states of excitons. The proposed design is an initial blueprint and is amenable to further optimization. For example, one ${ }^{28}$ or both of $\mathrm{MoS}_{2}$ layers can be substituted by a different transition metal dichalcogenide (TMD), such as $\mathrm{WS}_{2}$ or $\mathrm{WSe}_{2}$. The two $\mathrm{MoS}_{2}$ monolayers may be replaced by a single several-monolayer-thick TMD encapsulated by hBN in which indirect excitons would be composed of electrons and holes confined at the opposite sides of the TMD layer. The outlined principle for realizing high-temperature superfluidity can also be extended to layered materials other than TMD and $\mathrm{hBN}$.

Besides providing a new platform for exploring fundamental quantum phenomena, indirect excitons in van der Waals structures can be also utilized for the development of optoelectronic circuits ${ }^{4}$. In such devices, in-plane potential landscapes for excitons are created and controlled by external electric fields that couple to the permanent dipole moment $p=e c$ of the indirect excitons. The operation temperature for excitonic circuits in the van der Waals structures is expected to exceed by an order of magnitude the $\sim 100 \mathrm{~K}_{\text {record }}{ }^{29}$ set by GaAs-based CQWs.

Finally, we note that Fig. 3 was constructed following the example of the Monte-Carlo simulations ${ }^{10-12}$ in which only exciton phases with two possible spin flavours were considered. In TMDs, spin and orbital degeneracies of indirect excitons may have a more intriguing structure. Such degeneracies can be controlled by strong spin-orbit coupling ${ }^{30}$, polarization of the excitation beam ${ }^{7,31}$ or many-body interactions ${ }^{32}$.

Experimental realization of superfluidity and counterflow superconductivity as well as excitonic circuits and spintronic/ valleytronic devices in atomically thin heterostructures may have far-reaching implications for science and technology.

\section{Methods}

Interlayer tunnelling. The action $S$ for tunnelling of quasiparticles across the hBN spacer can be estimated from the usual formula for the rectangular potential barrier of height $U_{\mathrm{b}}$ :

$$
S=2\left(c_{\mathrm{b}} / a_{\mathrm{B}}\right)\left(m_{\mathrm{b}} / m_{0}\right) U_{\mathrm{b}}^{1 / 2}(\mathrm{Ry}) .
$$

Here $c_{\mathrm{b}}=N c_{1}$ is the tunnelling length, $N$ is the number of hBN layers, $c_{1}=0.333 \mathrm{~nm}$ is the thickness of one hBN layer, $a_{\mathrm{B}}=0.0529 \mathrm{~nm}$ is the hydrogen Bohr radius and $m_{\mathrm{b}}$ is the effective carrier mass inside the barrier. If the chemical potential is in the middle of the hBN energy gap, we expect $U_{\mathrm{b}} \approx 3 \mathrm{eV}$. Assuming also $m_{\mathrm{b}} \approx 0.5$ in units of the bare electron mass, we obtain $S \approx 5 \mathrm{~N}$, which is in agreement with $S=4.6 \mathrm{~N}$ deduced from the tunnelling conductance measured in graphene/hBN/graphene structures ${ }^{15}$. In comparison, in GaAs/AlGaAs CQW structures with $m_{\mathrm{b}} \approx 0.35, U_{\mathrm{b}} \approx 0.15 \mathrm{eV}$ and $d_{\mathrm{b}}=4.0 \mathrm{~nm}$ we get $S=9.4$. Hence, for $N=2$ the tunnelling rate is comparable to that in the $\mathrm{GaAs}_{\mathrm{CQW}}{ }^{4,5}$. More detailed estimates would have to include the trapezoidal shape of the barrier (Fig. 1b) and precise nature of the band alignment in the TMD/hBN structures. Nevertheless, it is clear that by a minor adjustment of $N$ in the range from, say, 2 to 4 , sufficiently long interlayer tunnelling lifetimes can be achieved for indirect excitons.

Binding energy of indirect excitons. We model both the electron and the hole that compose an indirect exciton as 2D quantum particles confined in the midplanes of two separate $\mathrm{MoS}_{2}$ layers. The Schrödinger equation for the relative motion reads

$$
-\frac{\hbar^{2}}{2 \mu} \nabla^{2} \phi(\mathbf{r})+U(\mathbf{r}) \phi(\mathbf{r})=-E_{\text {ind }} \phi(\mathbf{r}),
$$

where $\mu$ is the reduced mass. We model the potential $U(\mathbf{r})$ of electron-hole Coulomb interaction using the continuum-medium electrostatics ${ }^{8}$. This simplified approach neglects frequency dependence of the dielectric functions of the materials involved. We approximate each of $\mathrm{MoS}_{2}$ layers as a uniaxial dielectric slab of thickness $c_{2}=0.312 \mathrm{~nm}$ with principal dielectric tensor components ${ }^{8} \epsilon_{2}^{\perp}=14.29$ and $\epsilon_{2}^{\|}=6.87$ in the directions perpendicular and parallel to the $z$ axis, respectively. In turn, the hBN spacer is modelled as a slab of thickness $N c_{1}$, $c_{1}=0.333 \mathrm{~nm}$, with the dielectric constants ${ }^{33} \epsilon_{2}^{\perp}=6.71$ and $\epsilon_{1}^{\|}=3.56$. We define the anisotropy parameter $\kappa_{j}$ and the effective dielectric constant $\epsilon_{j}$ of each material by

$$
\kappa_{j}=\sqrt{\epsilon_{j}^{\perp}} / \sqrt{\epsilon_{j}^{\|}}, \quad \epsilon_{j}=\sqrt{\epsilon_{j}^{\perp}} \sqrt{\epsilon_{j}^{\|}}, \quad j=1,2,
$$

so that $\kappa_{2}=1.44, \epsilon_{2}=9.91\left(\mathrm{MoS}_{2}\right)$ and $\kappa_{1}=1.37, \epsilon_{1}=4.89(\mathrm{hBN})$. The interaction potential can be found by solving the Poisson equation for this layered system, which is done by reducing it to a set of linear equations for the Fourier component $\tilde{U}(\mathbf{q})$ and using the inverse Fourier transform:

$$
U(\mathbf{r})=\int \frac{\mathrm{d}^{2} q}{(2 \pi)^{2}} e^{i \mathbf{q r}} \tilde{U}(\mathbf{q}) .
$$

By symmetry, $\tilde{U}(\mathbf{q})$ depends only on the magnitude $q=|\mathbf{q}|$ of vector $\mathbf{q}$ not on its direction. After simple algebra, we arrive at the result $U(q)=C(q) / D(q)$, where functions $C(q)$ and $D(q)$ are given by

$$
\begin{gathered}
C(q)=\frac{8 \pi e^{2}}{q} \epsilon_{1}\left[\left(\epsilon_{1}-\epsilon_{2}\right) e^{-c_{2} k_{2} / 2}-\left(\epsilon_{1}-\epsilon_{2}\right) e^{c_{2} k_{2} / 2}\right]^{2} e^{-c k_{1}}, \\
D(q)=\left[\left(\epsilon_{1}-\epsilon_{2}\right)^{2} e^{-c_{2} k_{2}}-\left(\epsilon_{1}+\epsilon_{2}\right)^{2} e^{c_{2} k_{2}}\right]^{2} \\
-4\left(\epsilon_{1}^{2}-\epsilon_{2}^{2}\right)^{2} e^{-2\left(c-c_{2}\right) k_{1}} \sinh ^{2} c_{2} k_{2} .
\end{gathered}
$$

Here we introduced the short-hand notations $c=N c_{1}+c_{2}$ (the centre-to-centre distance) and $k_{j}=\kappa_{j} q$ (the $z$-direction wavenumber of the evanescent Fourier harmonics $e^{i q r \pm k_{j} z}$ in medium $j$ ). From numerical calculations using equations (9)-(11) we found that potential $U(r)$ is accurately approximated by the following analytical expression:

$$
U(r)=\frac{e^{2}}{\epsilon_{1}} \frac{1}{\sqrt{r^{2}+d^{2}}}\left(1-\frac{A}{1+B r^{2}}\right), \quad d=\kappa_{1} c .
$$

With a suitable choice of coefficients $0<A<1$ and $B>0$ this form produces asymptotically exact results for $U(r)$ at both small and large $r$.

To solve equation (7), we discretized it on a real-space 2D grid (typically, $75 \times 75)$. The resultant linear eigenvalue problem was diagonalized by standard numerical methods yielding the binding energy $E_{\text {ind }}(N)$ and the gyration radius $r_{\mathrm{x}}(N)$ of indirect excitons. The latter is defined in terms of a normalized groundstate wavefunction $\phi(r)$ by means of the integral

$$
r_{\mathrm{x}}^{2}=\int r^{2} \phi^{2}(r) \mathrm{d}^{2} r
$$

The results are shown in Fig. 2. For $N=2$ we find $r_{\mathrm{x}}=2.50 \mathrm{~nm}=2.43 a_{\mathrm{x}}$ and $E_{\text {ind }}=87 \mathrm{meV} \approx 0.6 \mathrm{Ry}_{\mathrm{x}}$. This binding energy is an order of magnitude larger than $E_{\text {ind }}=4-10 \mathrm{meV}$ typical for excitons in GaAs/AlGaAs CQW structures $^{29,34}$.

Zero-temperature phases. It is instructive to complement the above discussion of the finite- $T$ phase diagram (Fig. 3) with commenting on the $T=0$ phases. Such phases include electron-hole Fermi gas, exciton Bose gas and exciton solid. The approximate phase boundaries based on available Monte-Carlo calculations $^{10-12,35,36}$ are shown in Fig. 4. Although those simulations were done for electron-hole bilayers in vacuum, an approximate correspondence with our dielectric environment can be achieved if we neglect the correction term in the 
parenthesis in equation (12). The mapping is then obtained by setting the distance between the layers to $d=\kappa_{1} c$ and including the effective dielectric function $\epsilon_{1}$ in the definition of the electron Bohr radius:

$$
a_{\mathrm{e}}=\frac{\hbar^{2} \epsilon_{1}}{m_{\mathrm{e}} e^{2}}=\frac{1}{2} \frac{\hbar^{2} \epsilon_{1}}{\mu e^{2}}=\frac{a_{\mathrm{x}}}{2} .
$$

On the horizontal axis in Fig. 4 we plot the dimensionless intralayer distance parameter $r_{\mathrm{s}}=1 / \sqrt{\pi n_{\mathrm{e}} a_{\mathrm{e}}^{2}}$, where $n_{\mathrm{e}}$ is the total electron density. Solid phases form at large $r_{\mathrm{s}}$ and $d / a_{\mathrm{e}}$. The exciton dipole solid crosses over to the interlocked Wigner crystals of electrons and holes as the interlayer distance becomes larger than the characteristic intralayer one, $d \gtrsim r_{\mathrm{s}} a_{\mathrm{e}}$

The considered $\mathrm{MoS}_{2} / \mathrm{hBN}$ heterostructure with $N=2$ corresponds to $d / a_{\mathrm{e}} \approx 2.8$ for which the ground state is never a solid phase. Instead, at large $r_{\mathrm{s}}$, that is, at low electron density, the ground state of the system is an exciton gas. As $r_{\mathrm{s}}$ decreases, the Mott transition to electron-hole Fermi gas occurs. At this transition the excitons dissociate owing to screening and phase space filling ${ }^{1}$. The latest estimates $^{12}$ of the $T=0$ Mott transition at $d=2.8 a_{\mathrm{e}}$ give $r_{\mathrm{s}} \approx 6$, which corresponds to equation (3). Interestingly, a Bardeen-Cooper-Schrieffer-like excitonic state of a dense electron-hole gas predicted by the earlier theory ${ }^{37}$ was not found in the cited Monte-Carlo calculations ${ }^{10,12}$.

Unless the effective interlayer separation $d$ is small, the interaction between indirect excitons is dominated by the classical dipole repulsion term ${ }^{38,39}$. However, as shown previously $\mathrm{y}^{40-42}$, at $d<0.87 a_{\mathrm{e}}$ quantum exchange-correlation effects cause the change of repulsion to attraction leading to appearance of bi-excitons phases ${ }^{12,39}$. Narrow ranges of modulated phases (stripes, bubbles or supersolids) may exist near any of the first-order phase transition lines ${ }^{43}$.

\section{References}

1. Keldysh, L. V. \& Kozlov, A. N. Collective properties of excitons in semiconductors. J. Exp. Theor. Phys. 27, 521 (1968).

2. Lozovik, Y. E. \& Yudson, V. I. A new mechanism for superconductivity: pairing between spatially separated electrons and holes. J. Exp. Theor. Phys. 44, 389 (1976).

3. Fukuzawa, T., Kano, S., Gustafson, T. \& Ogawa, T. Possibility of coherent light emission from Bose condensed states of SEHPs. Surf. Sci. 228, 482-485 (1990).

4. High, A. A., Novitskaya, E. E., Butov, L. V., Hanson, M. \& Gossard, A. C. Control of exciton fluxes in an excitonic integrated circuit. Science 321, 229-231 (2008).

5. High, A. A. et al. Spontaneous coherence in a cold exciton gas. Nature 483, 584-588 (2012).

6. Geim, A. K. \& Grigorieva, I. V. Van der Waals heterostructures. Nature 499, 419-425 (2013).

7. Xiao, D., Liu, G.-B., Feng, W., Xu, X. \& Yao, W. Coupled spin and valley physics in monolayers of $\mathrm{MoS}_{2}$ and other group-VI dichalcogenides. Phys. Rev. Lett. 108, 196802 (2012).

8. Berkelbach, T. C., Hybertsen, M. S. \& Reichman, D. R. Theory of neutral and charged excitons in monolayer transition metal dichalcogenides. Phys. Rev. B 88, 045318 (2013).

9. Zhang, Y. et al. Direct observation of the transition from indirect to direct bandgap in atomically thin epitaxial $\mathrm{MoSe}_{2}$. Nat. Nanotechnol. 9, 111-115 (2013)

10. De Palo, S., Rapisarda, F. \& Senatore, G. Excitonic condensation in a symmetric electron-hole bilayer. Phys. Rev. Lett. 88, 206401 (2002).

11. Schleede, J., Filinov, A., Bonitz, M. \& Fehske, H. Phase diagram of bilayer electron-hole plasmas. Contrib. Plasma Phys. 52, 819-826 (2012).

12. Maezono, R., López Ros, P., Ogawa, T. \& Needs, R. J. Excitons and biexcitons in symmetric electron-hole bilayers. Phys. Rev. Lett. 110, 216407 (2013).

13. Mak, K. F., Lee, C., Hone, J., Shan, J. \& Heinz, T. F. Atomically thin $\mathrm{MoS}_{2}$ : a new direct-gap semiconductor. Phys. Rev. Lett. 105, 136805 (2010).

14. Chernikov, A. et al. Non-hydrogenic exciton Rydberg series in monolayer $\mathrm{WS}_{2}$. Preprint at http://arxiv.org/abs/1403.4270 (2014).

15. Britnell, L. et al. Field-Effect tunneling transistor based on vertical graphene heterostructures. Science 335, 947-950 (2012).

16. Nikolaev, V. V. \& Portnoi, M. E. Mott transition of spatially indirect excitons. Proc. SPIE 5509, 187-193 (2004).

17. Butov, L. V., Kulakovskii, V. D., Lach, E., Forchel, A. \& Grützmacher, D. Magnetoluminescence study of many-body effects in homogeneous quasi-twodimensional electron-hole plasma in undoped $\operatorname{In}_{\mathrm{x}} \mathrm{Ga}_{1-x} \mathrm{As} / \mathrm{InP}$ single quantum wells. Phys. Rev. B 44, 10680-10688 (1991).

18. Kappei, L., Szczytko, J., Morier-Genoud, F. \& Deveaud, B. Direct observation of the Mott transition in an optically excited semiconductor quantum well. Phys. Rev. Lett. 94, 147403 (2005).

19. Filinov, A., Prokof ev, N. V. \& Bonitz, M. Berezinskii-Kosterlitz-Thouless transition in two-dimensional dipole systems. Phys. Rev. Lett. 105, 070401 (2010)
20. Su, J.-J. \& MacDonald, A. H. How to make a bilayer exciton condensate flow. Nat. Phys. 4, 799-802 (2008)

21. Littlewood, P. B. et al. Models of coherent exciton condensation. J. Phys. Condens. Matter 16, S3597-S3620 (2004).

22. Eisenstein, J. P. \& MacDonald, A. H. Bose-Einstein condensation of excitons in bilayer electron systems. Nature 432, 691-694 (2004).

23. McCann, E. \& Koshino, M. The electronic properties of bilayer graphene. Rep. Prog. Phys. 76, 056503 (2013).

24. Min, H., Bistritzer, R., Su, J.-J. \& MacDonald, A. H. Room-temperature superfluidity in graphene bilayers. Phys. Rev. B 78, 121401 (2008).

25. Perali, A., Neilson, D. \& Hamilton, A. R. High-temperature superfluidity in double-bilayer graphene. Phys. Rev. Lett. 110, 146803 (2013).

26. Neilson, D., Perali, A. \& Hamilton, A. R. Excitonic superfluidity and screening in electron-hole bilayer systems. Phys. Rev. B 89, 060502(R) (2014).

27. Kharitonov, M. Y. \& Efetov, K. B. Electron screening and excitonic condensation in double-layer graphene systems. Phys. Rev. B 78, 241401 (2008).

28. Komsa, H.-P. \& Krasheninnikov, A. V. Electronic structures and optical properties of realistic transition metal dichalcogenide heterostructures from first principles. Phys. Rev. B 88, 085318 (2013).

29. Grosso, G. et al. Excitonic switches operating at around 100 K. Nat. Photonics 3 , 577-580 (2009)

30. Kormányos, A., Zólyomi, V., Drummond, N. D. \& Burkard, G. Spin-orbit coupling, quantum dots, and qubits in monolayer transition metal dichalcogenides. Phys. Rev. X 4, 011034 (2014).

31. Ross, J. S. et al. Electrical control of neutral and charged excitons in a monolayer semiconductor. Nat. Commun. 4, 1474 (2013).

32. Ben-Tabou de Leon, S. \& Laikhtman, B. Mott transition, biexciton crossover, and spin ordering in the exciton gas in quantum wells. Phys. Rev. B 67, 235315 (2003).

33. Cai, Y., Zhang, L., Zeng, Q., Cheng, L. \& Xu, Y. Infrared reflectance spectrum of BN calculated from first principles. Solid State Commun. 141, 262-266 (2007).

34. Szymanska, M. H. \& Littlewood, P. B. Excitonic binding in coupled quantum wells. Phys. Rev. B 67, 193305 (2003).

35. Astrakharchik, G. E., Boronat, J., Kurbakov, I. L. \& Lozovik, Y. E. Quantum phase transition in a two-dimensional system of dipoles. Phys. Rev. Lett. 98, 060405 (2007)

36. Büchler, H. P. et al. Strongly correlated 2D quantum phases with cold polar molecules: controlling the shape of the interaction potential. Phys. Rev. Lett. 98, 060404 (2007).

37. Keldysh, L. V. \& Kopaev, Y. V. Possible instability of the semimetallic state toward Coulomb interaction. Sov. Phys. Solid State 6, 2219 (1965).

38. Zhu, X., Littlewood, P. B., Hybertsen, M. S. \& Rice, T. M. Exciton condensate in semiconductor quantum well structures. Phys. Rev. Lett. 74, 1633-1636 (1995)

39. Lozovik, Y. E. \& Berman, O. L. Phase transitions in a system of two coupled quantum wells. JETP Lett. 64, 573-579 (1996).

40. Meyertholen, A. D. \& Fogler, M. M. Biexcitons in two-dimensional systems with spatially separated electrons and holes. Phys. Rev. B 78, 235307 (2008).

41. Tan, M. Y. J., Drummond, N. D. \& Needs, R. J. Exciton and biexciton energies in bilayer systems. Phys. Rev. B 71, 033303 (2005).

42. Schindler, C. \& Zimmermann, R. Analysis of the exciton-exciton interaction in semiconductor quantum wells. Phys. Rev. B 78, 045313 (2008).

43. Spivak, B. \& Kivelson, S. A. Phases intermediate between a two-dimensional electron liquid and Wigner crystal. Phys. Rev. B 70, 155114 (2004).

\section{Acknowledgements}

This work was supported by the US ONR \& UCOP (M.M.F.), NSF (L.V.B.) and also by the European Research Council and EC-FET European Graphene Flagship (K.S.N.). We are grateful to André K. Geim for comments.

\section{Author contributions}

All the authors contributed to the ideas, discussion, and writing of the paper. M.M.F. carried out numerical simulations.

\section{Additional information}

Competing financial interests: The authors declare no competing financial interests.

Reprints and permission information is available online at http://npg.nature.com/ reprintsandpermissions/

How to cite this article: Fogler, M. M. et al. High-temperature superfluidity with indirect excitons in van der Waals heterostructures. Nat. Commun. 5:4555 doi: $10.1038 /$ ncomms5555 (2014) 